\title{
Type 2 immunity at the origin of beige adipocytes
}

T

he development of beige adipose tissue is mediated in large measure by innate immune cells and molecules, reveal two independent studies in mice published in the same issue of Cell.

Brown adipose tissue (BAT) increases energy expenditure via heat dissipation in response to cold exposure or exercise. In classic brown adipocytes-which are present in rodents and human infants, but not in human adults-this thermogenic process is activated by pathways involving the central nervous system and the sympathetic nervous system. Beige adipocytes are brown-like adipocytes that exist within white adipose tissue (WAT) in adult humans, but how thermogenesis is triggered in beige adipocytes was unclear.

Bruce Spiegelman and colleagues used a combination of proteomic and genomic approaches to identify a hormone, meteorin-like (Metrnl), that is a target of PGC-1 14 , a protein involved in the response of skeletal muscle to exercise. Metrnl expression increased in muscle after exercise and in adipose tissue after cold exposure. Increased expression of Metrnl was associated with increased energy expenditure and improved glucose tolerance in obese and diabetic mice. Experiments using an adenoviral vector showed that Metrnl stimulates the accumulation of eosinophils that express IL- 4 in adipose tissue, which then leads to alternative activation of macrophages. These macrophages were shown to be required for thermogenesis and production of anti-inflammatory factors in adipose tissue. Use of Metrnl blockers inhibited the

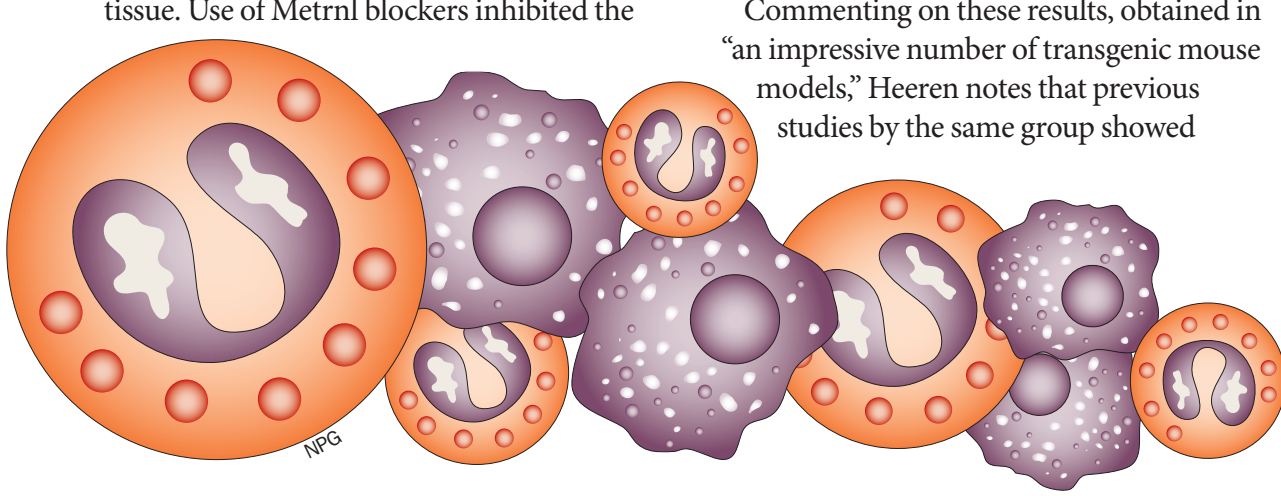

identified signalling cascade and its effects. "This is an unusual mechanism whereby a new factor made by muscle causes browning of fat," sums up Spiegelman.

"In contrast to endocrine factors, such as FGF21, known to directly promote beige adipocyte development, the signalling cascade mediated by Metrnl provides a novel and surprising mechanism in the complex hormonal and cellular network regulating the browning of WAT," comments Jörg Heeren (University of Hamburg, Germany), who was not involved in either of the two studies.

Ajay Chawla and colleagues observed that cold exposure stimulated activation of eosinophils that produce IL-4 and IL-13. These cytokines triggered alternative activation of macrophages via IL-4 receptor $\alpha$ and STAT6. Expression of tyrosine hydroxylase and catecholamine production were increased in these macrophages, which then led to browning of adipose tissue. "We demonstrated that type 2 innate immunity was absolutely necessary for development of coldinduced beige fat," says Chawla, "and also that catecholamine production by M2 macrophages rather than sympathetic nerves was required for beige fat thermogenesis." Administration of IL-4 to mice that were not experiencing cold stress was sufficient to induce development of beige adipose tissue. Finally, induction of beige adipocytes was sufficient to reduce adiposity and improve metabolic dysfunction in a mouse model of pre-established obesity.

Commenting on these results, obtained in impressive number of transgenic mouse models," Heeren notes that previous studies by the same group showed that IL-4-activated macrophages sustained adaptive thermogenesis in BAT and that in the absence of a functional IL-4/STAT6 axis the body core temperature severely declined during a cold challenge. "Thus, it is somehow surprising that in the current study, adaptive thermogenesis mediated by BAT is apparently unaffected by the absence of either eosinophils or macrophages after long-term cold activation. The immediate increases in energy expenditure observed in the current study in response to cold suggest that there is also an important contribution by classic brown adipocytes." Chawla clarifies that the previously reported acute defect in IL-4/IL-13 and STAT6 knockout mice results from impaired lipolysis of stored triglycerides in WAT. "While this defect contributes to acute impairment of brown fat activity, long-term adaptation requires recruitment of beige adipocytes in WAT, which is dependent on the same type 2 immune pathway."

"Our study provides rigorous proof for targeting beige fat to mitigate obesity and its metabolic complications," says Chawla. "In addition, it suggests that type 2 immunity is the dominant pathway controlling beige fat development, providing new cellular and molecular targets." Spiegelman is also interested in the therapeutic implications of the findings, not only in the context of obesity and diabetes mellitus but also in the context of muscle disease-for example in muscular dystrophy and other types of muscle damage. "The mechanisms described in the two publications carry great therapeutic potential for the treatment of obesity and its associated comorbidities," concurs Heeren, "with the caveat that manipulations of the immune system are potentially risky."

\section{Joana Osório}

Original articles Rao, R. R. et al. Meteorin-like is a hormone that regulates immune-adipose interactions to increase beige fat thermogenesis. Cell 157, 1279-1291 (2014) | Qiu, Y. et al. Eosinophils and type 2 cytokine signaling in macrophages orchestrate development of functional beige fat. Cell 157, 1292-1308 (2014) 